\title{
Ultrasound Guided Platelet Rich Plasma Injection in Patients with Primary Knee Osteoarthritis
}

\author{
Ali Eed El-Deeb, Amal Mohamad EL-Barbary, Radwa Mostafa El Khouly, Nevien Ibrahim Elfeky * \\ Department of Rheumatology and Rehabilitation, Faculty of Medicine, Tanta University \\ *Correspondence author: Nevien Ibrahim Elfeky, Mobile: (+20)01126726758, E-mail: nova_elfeky87@yahoo.com
}

\begin{abstract}
Background: Osteoarthritis is a leading cause of pain, disability, and socioeconomic cost worldwide and the knee is one of the most commonly affected joint.

Objective: to evaluate clinical and functional therapeutic effect of ultrasound guided intraarticular or periarticular platelet rich plasma (PRP) injection in treatment of patients with primary knee osteoarthritis.

Patients and Methods: eighty patients with primary knee osteoarthritis were divided into two groups: Group I: 50 knees, group II: 30 knees. Both groups were treated by PRP injection into the lateral recess of the knee group I or into painful periarticular structure that have previously been identified on ultrasound group II.

Results: The VAS in group I $(6.3 \pm 1.45)$ and II $(6.57 \pm 1.30)$ significantly improved after injection $(1.30 \pm 0.97$ and $1.43 \pm 0.77 ; \mathrm{p}<0.001)$. The range of movement $(\mathrm{ROM})$ (flexion) significantly improved in both groups after injection. The WOMAC significantly improved in both groups after injection ( $<<0.001$ ); (group I: $14.04 \pm 7.73$ vs group II: $15.20 \pm 7.12)$. The KOOS score significantly improved in group I $(41.93 \pm 11.05$ to $75.43 \pm 7.70 ; \mathrm{p}<0.001)$ and group II $(39.83 \pm 12.46$ to $76.08 \pm 7.12 ; \mathrm{p}<0.001)$ after injection. There was a significant improvement in the percentage of different parameters in mild and moderate knee osteoarthritis in both groups.

Conclusion: Ultrasound guided single dose PRP injection is a safe and effective method for treating all grades of knee OA mainly mild to moderate also injection in periarticular pain generator structures reduce pain and improve knee function.
\end{abstract}

Keywords: Knee, Osteoarthritis, Ultrasound-guided injection, Platelet rich plasma.

\section{INTRODUCTION}

Osteoarthritis (OA) is a leading cause of pain, disability, and socioeconomic cost worldwide and the knee is one of the most commonly affected joint. The epidemiology of the disorder is multifactorial, with genetic, biological, etiological and biomechanical components $^{(1)}$.

The clinical diagnosis of primary knee osteoarthritis is based on patient history and physical examination and radiological findings. Ultrasound has proven to be a noninvasive, safe and is more sensitive than clinical examination for diagnosing pathologic conditions of the knee joint, including the tendons, ligaments, muscles, synovial fluid, articular cartilage, nerves, and surrounding soft tissues. In addition; color and power Doppler techniques can be used to measure neovascularization of the synovial joints lining, tendons, and soft-tissue masses ${ }^{(2-4)}$. Ultrasonic guidance injection can be a valid tool to select a proper injection site, to increase the efficacy and reduce complications of blind injection ${ }^{(5)}$. Ultrasound-guided injection was effective in different lesions as plantar fasciitis ${ }^{(6)}$ and rotator cuff tendinopathy ${ }^{(7)}$.

Treatment of primary knee OA aims to relieve pain and restore knee function. Conventional therapies include activity modification, oral medication, nutrition, physical therapy and joint injection ${ }^{(8)}$. The use of PRP as a biological solution for pain relief and functional improvements in patients with primary knee OA has gained popularity over the last several years.
Ultrasound-guided PRP injections have become a quick and safe procedure ${ }^{(6)}$.

PRP is a whole blood fraction containing high platelet concentrations. Platelets are closely involved in hemostasis, inflammation, innate and adaptive immunity, tissue regeneration and other physiological and pathological processes. Platelets enclose dense granules that release a wide range of bioactive substances in response to agonists. When activated, platelet membranes release thrombotic and inflammatory agents, which may take an active part in the pathophysiology of autoimmune and autoinflammatory disorders ${ }^{(9)}$. In addition to various growth factors which participate in tissue repair processes to accelerate the healing response and to enhance the regeneration of bones and soft tissues ${ }^{(10,11)}$.

The aim of this study was to evaluate clinical and functional therapeutic effect of intraarticular or periarticular PRP injection guided by ultrasound in treatment of patients with primary knee osteoarthritis.

\section{AIM OF THE WORK}

To evaluate clinical and functional therapeutic effect of ultrasound guided intraarticular or periarticular platelet rich plasma (PRP) injection in treatment of patients with primary knee osteoarthritis.

\section{PATIENTS AND METHODS:}

Inclusion criteria: This randomized control study was carried out on 80 knees of 80 patients with primary knee osteoarthritis selected from the Outpatient Clinic of 
Physical Medicine, Rheumatology and Rehabilitation, Tanta University Hospitals. They were diagnosed according to the criteria of the American College of Rheumatology ${ }^{(12)}$.

Exclusion criteria: Patients with body mass index more than 40, previous injection in the knee within last 6 months, patients who are suffering from diabetes mellitus, presence of tumors or metastasis and immune deficiencies, active infection in the area to be treated, low platelet count, anemia and thrombocytopenia, and recent nonsteroidal antiinflammatory drugs (NSAIDs) use in the last week, and patients who can't comply with post procedure guidelines were excluded from the study.

All patients gave their informed consent prior to their inclusion and the study was approved by the Ethical Committee of Tanta University Hospitals in accordance to the declaration of Helsinki.

Clinical assessment: pain by (VAS) at rest ${ }^{(13)}$, morning stiffness in minutes, joint line tenderness (0-absent, 1present) and goniometric assessment of active and passive knee joint $\mathrm{ROM}^{\left({ }^{(14)} \text {. }\right.}$

Functional assessment: Western Ontario McMaster Universities index (WOMAC) its consists of 24 items divided into 3 subscales: 1-Pain subscale: 2-Stiffness Subscale: 3-Function Subscale ${ }^{(15)}$.

Knee injury and osteoarthritis outcome score (KOOS): It is a knee-specific instrument, developed to detect improvement or deterioration in symptoms, function, and sports activities due to knee impairment. It covers 42 items in 5 separately scored subscales; pain, other symptoms, function in daily living (ADL), function in sport and recreation (Sport/Rec), and knee-related quality of life (QOL). Scoring items are scored from 0 (no problems) to 4 (extreme problems). The total score is calculated as (sum of items)/(maximum possible score $) \times 100$, to give a total score of 100 where $100=$ no limitation with daily or sporting activities and the absence of symptoms according to the equation (16).

Ultrasonographic assessment: all patients underwent sonographic examination at the Ultrasound Unit of Physical Medicine, Rheumatology and Rehabilitation Department of Tanta University Educational Hospital by SAMSUNG MEDISON (UGEOH60) machine and using linear array transducers with frequencies ranging between 9 and $13 \mathrm{MHz}$. The ultrasonographic findings of the patients were recorded before and 6 weeks after injection according to US scored by Catay et al. ${ }^{(17)}$ US score of knee osteoarthritis includes variables points: Joint effusion if present 1, Degenerative femoral hyaline cartilage involvement if present 4, Osteophytes if present 3, Menisci protrusion if present 2, maximum total score 10.

X-ray radiographic assessment: The severity of knee OA was graded on knee radiographs according to the Kellgren-Lawrence (K. and L.) scale ( 0 , normal, I: small osteophytes without clinical importance; II: definite osteophytes but normal joint space; III: definite osteophytes with moderate narrowing of joint space; IV: definite osteophytes with severe narrowing of joint space). Patients with knee OA grades II-IV were included ${ }^{(18)}$.

\section{Study design:}

The selected patients were divided according to the cause of pain (intraarticular or periarticular) diagnosed clinically and by ultrasound into two groups: Group I: 50 patients were injected by $4 \mathrm{ml}$ of PRP into the lateral recess of the knee. Group II: 30 patients were injected by $4 \mathrm{ml}$ of PRP into painful periarticular structure (iliotibial band, lateral collateral ligament, medial collateral ligament and patellar tendon) that have previously been identified on ultrasound.

Instruction after injection: no weight bearing or protected weight bearing with relative rest and gentle active range of motion ${ }^{(19)}$ during the first 3 days after injection. Paracetamol and cold compression were allowed if needed for control of post-injection pain. Patients progress to full weight bearing with gentle, prolonged stretching exercise to the muscles around the knee 4 to 14 days after injection. From third to six weeks, patients begin low intensity strengthening exercises (low weight, high repetition isotonic exercises) ${ }^{(20)}$.

PRP Preparation: PRP (40 ml of venous blood was drawn from each patient into vacuum tubes containing 4 $\mathrm{ml}$ of $10 \%$ sodium citrate). PRP was prepared according to a double-centrifugation protocol, first centrifugation: The tubes were centrifuged at $1000 \mathrm{rpm}$ for $15-20 \mathrm{~min}$ to create upper plasma layer, middle buffy coat layer and a lower red blood cell layer. The upper and middle layers were collected in a sterile empty tube and secondary recentrifuged at $3500 \mathrm{rpm}$ for $10 \mathrm{~min}$ resulting in the formation of a platelet plug and platelet-poor plasma (ppp). Platelet-poor plasma was withdrawn and discarded. The remaining PRP and platelet plug were reconstituted using gentle manual agitation resulting in $4 \mathrm{ml}$ of PRP ready for injection. Thrombin was added to activate PRP in ratio 10:1, it was directly mixed prior to injection. PRP should be injected within 30 minutes after preparation ${ }^{(21)}$. Figure (1) 


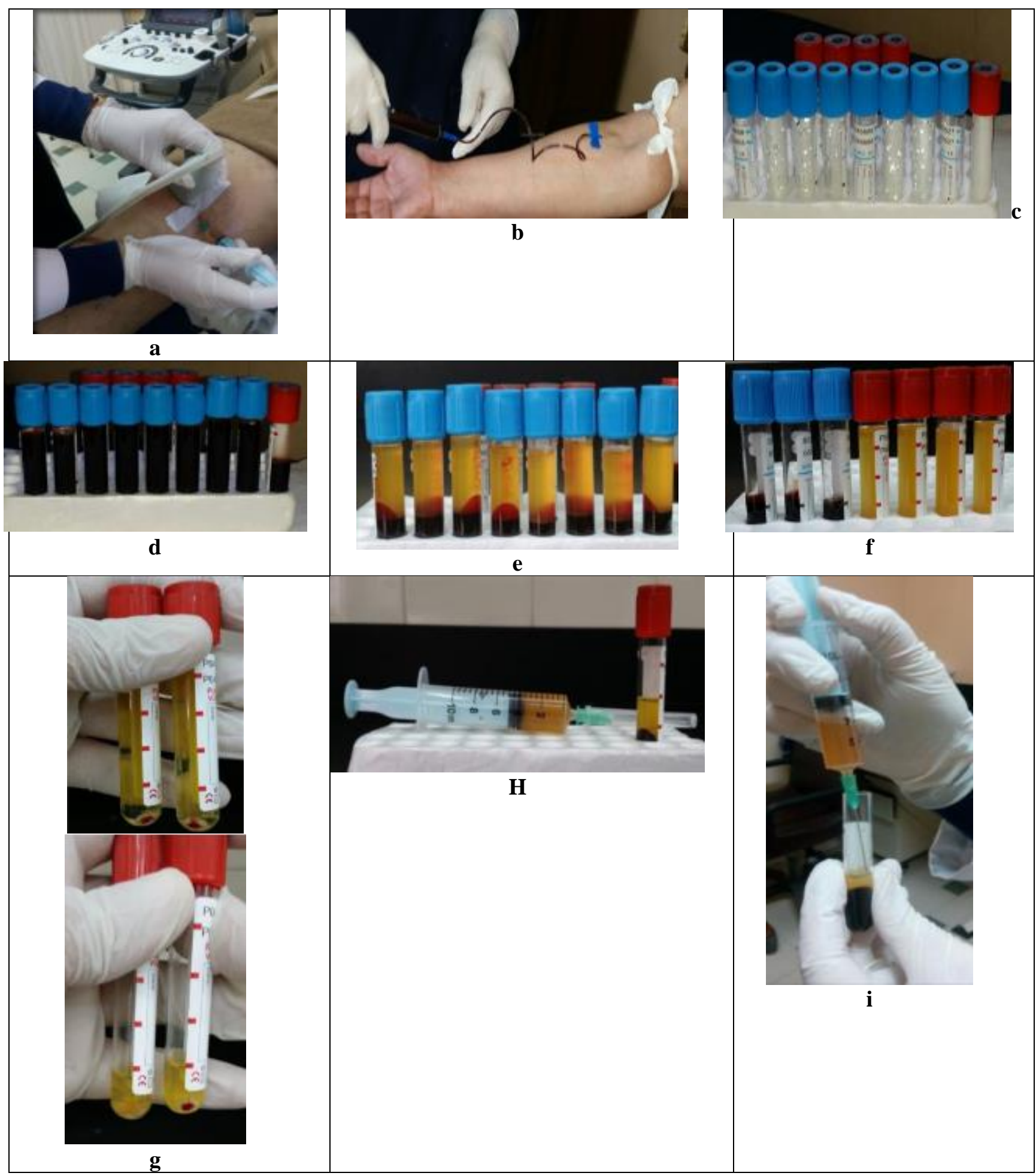

Figure (1): Preparation of PRP: A) Venipuncture from the patient. B) Vacuum tubes containing anticoagulant plus one without anticoagulant. C) Tubes after blood collection and before centrifugation. D) Tubes after the $1^{\text {st }}$ centrifugation. E) Plasma transferred to sterile tubes without anticoagulant before the $2^{\text {nd }}$ centrifugation. F) Plasma tubes with platelet pellet, after the second centrifugation. G) PRP after ppp withdrawal H) PRP stored in a $10 \mathrm{ml}$ syringe and blood with no anticoagulant after centrifugation, in order to obtain autologous thrombin. I) Activation of PRP with the autologous thrombin. 
ejhm.journals.ekb.eg

\section{Statistical analysis}

Recorded data were analyzed using the statistical package for social sciences, version 20.0 (SPSS Inc., Chicago, Illinois, USA). Quantitative data were expressed as mean \pm standard deviation (SD). Qualitative data were expressed as frequency and percentage.

\section{The following tests were done:}

- Independent-samples t-test of significance was used when comparing between two means.

- Chi-square $\left(\mathrm{x}^{2}\right)$ test of significance was used in order to compare proportions between two qualitative parameters.

- The confidence interval was set to $95 \%$ and the margin of error accepted was set to $5 \%$. The p-value was considered significant as the following:
- Probability (P-value)

P-value $<0.05$ was considered significant.

P-value $<0.001$ was considered as highly significant.

$\mathrm{P}$-value $>0.05$ was considered insignificant.

\section{RESULTS}

In the present work, regarding demographic data, patients receiving intraarticular PRP in group I had a mean age which was comparable to the age of patients receiving periarticular PRP in group II. Sex was matched in both groups. The right side was involved in $29(58 \%)$ patients in group I and 9 (30\%) in group II (Table 1).

Table (1): Comparison between the two studied groups according to demographic data, disease duration, sidedness and plain radiography $(\mathrm{K}$. and L.) in primary knee osteoarthritis patients.

\begin{tabular}{|c|c|c|c|}
\hline & $\begin{array}{l}\text { Group I } \\
(\mathbf{n}=\mathbf{5 0})\end{array}$ & $\begin{array}{c}\text { Group II } \\
(\mathbf{n}=\mathbf{3 0})\end{array}$ & $\mathbf{p}$ \\
\hline $\begin{array}{l}\text { Age (years) } \\
\text { Min. - Max. } \\
\text { Mean } \pm \text { SD. }\end{array}$ & $\begin{array}{l}46.0-66.0 \\
52.92 \pm 5.10(45.0-66.0)\end{array}$ & $\begin{array}{l}45.0-65.0 \\
53.70 \pm 4.14(48.0-65.0)\end{array}$ & 0.480 \\
\hline Sex (M:F) & $6: 44$ & $3: 27$ & 1.000 \\
\hline $\begin{array}{l}\text { Duration (years) } \\
\text { Min. - Max. } \\
\text { Mean } \pm \text { SD. }\end{array}$ & $\begin{array}{l}1.50-12.0 \\
5.64 \pm 2.68\end{array}$ & $\begin{array}{l}2.0-11.0 \\
6.03 \pm 2.30\end{array}$ & 0.389 \\
\hline $\begin{array}{l}\text { Body mass index } \\
\left(\mathrm{kg} / \mathrm{m}^{2}\right) \\
\text { Min. - Max. } \\
\text { Mean } \pm \text { SD. }\end{array}$ & $\begin{array}{l}25.0-39.80 \\
35.48 \pm 3.92 \\
\end{array}$ & $\begin{array}{c}28.10-39.70 \\
34.43 \pm 3.55 \\
\end{array}$ & 0.237 \\
\hline Affected knee (Rt:Lt) & $29: 21(29+21=60$ Not 50??) & $9: 21$ & $0.015^{*}$ \\
\hline $\begin{array}{l}\text { Plain radiography (K. } \\
\text { and L.) } \\
\text { Grade II } \\
\text { Grade III } \\
\text { Grade IV }\end{array}$ & $\begin{array}{l}\text { No } \\
11 \\
25 \\
14\end{array}$ & $\begin{array}{l}\text { No } \\
8 \\
13 \\
9\end{array}$ & 0.830 \\
\hline
\end{tabular}

Group I = Intraarticular group, group II = Periarticular group.

Regarding clinical and functional assessment, six weeks post-injection, there was a significant improvement of pain, WOMAC, KOOS, ROM and joint line tenderness in each group with insignificant difference between both groups (Table 2). 
Table (2): Comparison between the two studied groups according to different studied parameters before and 6 weeks after treatment in primary knee osteoarthritis patients.

\begin{tabular}{|c|c|c|c|c|}
\hline & $\begin{array}{c}\text { Parameters } \\
\text { fore/after injection } \\
\text { Mean } \pm \text { SD }\end{array}$ & $\begin{array}{l}\text { Group I } \\
(n=50)\end{array}$ & $\begin{array}{l}\text { Group II } \\
(\mathbf{n}=\mathbf{3 0})\end{array}$ & $\mathbf{P}$ \\
\hline \multirow[t]{3}{*}{ AS } & efore & $6.32 \pm 1.45$ & $6.57 \pm 1.30$ & 626 \\
\hline & fter & $1.30 \pm 0.97$ & $1.43 \pm 0.77$ & 486 \\
\hline & & $5.38 \times 10^{-10}$ & $1.41 \times 10^{-6}$ & \\
\hline \multirow[t]{3}{*}{ orning stiffness } & fore & $5.50 \pm 4.47$ & $6.53 \pm 4.20$ & 225 \\
\hline & fter & $0.86 \pm 1.43$ & $1.0 \pm 1.29$ & 0.503 \\
\hline & & $22.22 \times 10^{-9}$ & $3.64 \times 10^{-6}$ & \\
\hline \multirow[t]{3}{*}{ OMAC } & efore & $50.26 \pm 13.35$ & $53.63 \pm 16.59$ & 360 \\
\hline & fter & $14.04 \pm 7.73$ & $15.20 \pm 7.12$ & 360 \\
\hline & & $7.45 \times 10^{-10}$ & $1.72 \times 10^{-6}$ & \\
\hline \multirow[t]{3}{*}{$\mathrm{OOS}$} & efore & $41.93 \pm 11.05$ & $39.83 \pm 12.46$ & 735 \\
\hline & fter & $75.43 \pm 7.70$ & $76.08 \pm 7.12$ & 968 \\
\hline & & $7.55 \times 10^{-10}$ & $1.73 \times 10^{-6}$ & \\
\hline \multirow{6}{*}{$\begin{array}{l}\text { ange of motion (ROM) } \\
\text { exion }\end{array}$} & ctive Before & $116.64 \pm 11.79$ & $117.0 \pm 9.25$ & 815 \\
\hline & fter & $125.10 \pm 9.01$ & $127.0 \pm 5.66$ & 458 \\
\hline & & $1.67 \times 10^{-8}$ & $4.35 \times 10^{-6}$ & \\
\hline & assive Before & $120.54 \pm 10.04$ & $120.17 \pm 7.82$ & 549 \\
\hline & fter & $127.20 \pm 8.58$ & $128.40 \pm 4.79$ & 813 \\
\hline & & $1.75 \times 10^{-7}$ & $4.16 \times 10^{-6}$ & \\
\hline \multirow[t]{3}{*}{ int line tenderness } & efore +ve & $100.0 \%$ & $83.3 \%$ & \\
\hline & fter $+v e$ & $10.0 \%$ & $3.3 \%$ & \\
\hline & & $1.97 \times 10^{-11}$ & $7.24 \times 10^{-8}$ & \\
\hline
\end{tabular}

Group I = Intraarticular group, group II = Periarticular group.

VAS: visual analogue scale; WOMAC: Western Ontario McMaster Universities index; KOOS: knee injury and osteoarthritis outcome score; ROM: range of motion.

In Our study no complications such as infection or fever occurred among studied patients. Only minor adverse events were detected in 5 patients such as mild pain at injected area and skin bruises.

Regarding ultrasound findings, causes of periarticular pain in our patients are shown in Table 3.

Table (3): Causes of periarticular pain according to ultrasound findings

\begin{tabular}{|l|l|l|}
\hline \multicolumn{1}{|c|}{ Group II (n= 30) } \\
\hline \multicolumn{1}{|c|}{ Cause } & No. & $\%$ \\
\hline otibial band & & $\mathbf{3 . 4}$ \\
\hline edial collateral ligament & & $\mathbf{3 . 3}$ \\
\hline ateral collateral ligament & & ) \\
\hline tellar tendon & & $\mathbf{3}$ \\
\hline
\end{tabular}

Group II = Periarticular group.

Ultrasonographic imaging of knee joint effusion before and after injection are shown Fig. 2 and 3. 
ejhm.journals.ekb.eg

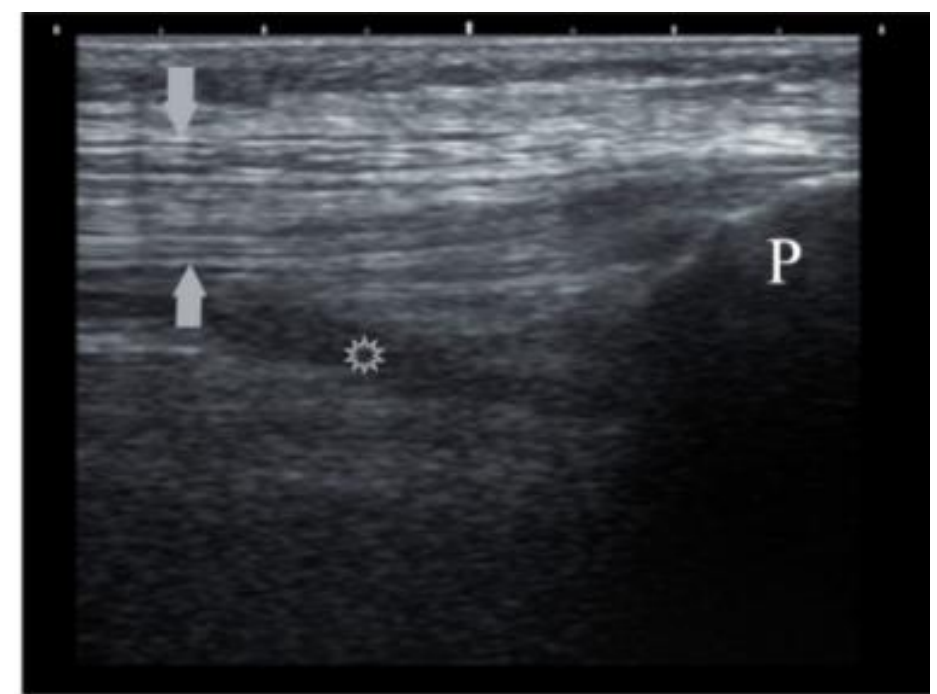

Figure (2): Long axis view of suprapatellar joint recess and quadriceps tendon with synovitis (effusion) before treatment [P: patella, F: femur, arrows: quadriceps tendon, star: sup-patellar recess].

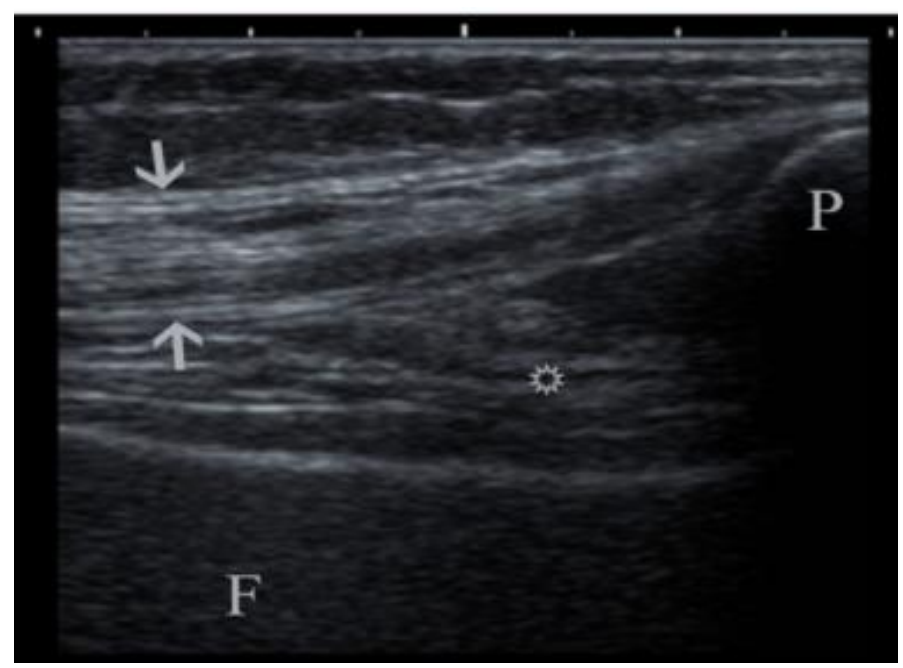

Figure (3): Long axis view of suprapatellar joint recess with decreased effusion and quadriceps tendon after treatment. [P: patella, F: femur, arrows: quadriceps tendon, star: sup-patellar recess].

\section{DISCUSSION}

Knee osteoarthritis is one of the most common progressive painful and disabling joint diseases worldwide and its treatment remains controversial ${ }^{(23,24)}$. Joint pain, stiffness and limitation of movement are the primary symptoms of $\mathrm{OA}^{(25)}$.

Some of the knee pain does not originate from the joint itself but relatively from the periarticular structures around the knee joint that contain pain fibers, including; patellar tendinopathy, friction of the iliotibial band (ITBF), contracture or stretch of medial collateral ligament (MCL) and lateral collateral ligament (LCL) and pressure of osteophytes against them and other capsular structures ${ }^{(26,27)}$.

PRP can delivers a natural concentrate of autologous blood growth factors which can be used for maintaining joint homeostasis, stimulation and acceleration of tissue healing and regeneration, including cartilage ${ }^{(28)}$.
Platelet rich plasma represent a major paradigm shift and advancement in the treatment of knee osteoarthritis when compared to intraarticular corticosteroid injections. However, there remains a need for strong, sufficiently powered, randomized controlled trials to validate its use over other traditional injection treatments (corticosteroid and viscosupplementation). Future research will need to optimize growth factor enhancement, consider the role of PRP alone and in combination with stem cells and scaffolds, as well as, determine optimal delivery method ${ }^{\mathbf{2 9} 9}$.

The studied patients with primary knee osteoarthritis in both groups had moderate to severe pain assessed by VAS at rest, morning stiffness, tenderness and limited ROM before injection, with significant improvement in both groups six weeks after treatment. These results were in agreement with Kon et al. ${ }^{(30)}$ and Gosens et al. ${ }^{(31)}$ who performed a study of single PRP injections into the site of patellar tendinopathy and found 
that all patients had statistically significant better pain relief in VAS at follow up.

A possible explanation for pain relief was related to platelet alpha granules which contain growth factors (platelet derived growth factor, insulin like growth factor, vascular endothelial growth factor, and transforming growth factor $\beta$ ) that are involved in each stage of the healing cascade. The injection of autologous PRP into the joint space and surrounding painful soft tissues delivers a concentrated dose of these growth factors, which enhance the healing process, decrease inflammation and reduce pain ${ }^{(32)}$.

Patients in both groups had severe functional limitation and disability as assessed by the WOMAC and KOOS scores and remarkably improved in both groups post-injection. These results agreed with Patel et al. ${ }^{(32)}$, Gobbi et al. ${ }^{(33)}$, and Paterson et al. ${ }^{(34)}$ who found in their studies that ROM, VAS, WOMAC and KOOS significantly improved at follow up in knee OA patients treated with PRP injection. Furthermore, single injection of PRP was as effective as 2 injections to alleviate symptoms in early knee $\mathrm{OA}^{\left({ }^{(33)} \text {. }\right.}$

According to the ultrasonic assessment before and six weeks after treatment in both groups, we used US scored by Catay $\boldsymbol{e t}$ al. ${ }^{(\mathbf{1 7})}$ to assess intraarticular; joint effusion, degenerative femoral hyaline cartilage involvement, osteophytes and menisci protrusion, and periarticular; iliotibial band, medial collateral ligament, lateral collateral ligament and patellar tendon lesions. There was statistically significant improvement in total US score in both groups and there was significant improvement of knee effusion in group I only after treatment compared with US score before treatment.

In our study both groups; intraarticular and periarticular injection was done guided by US to allow accurate injection site. This is in agreement with Huang et $\boldsymbol{a l} .{ }^{(35)}$ who also performed a study to evaluate the effectiveness and accuracy of ultrasound-guided intraarticular and periarticular joint injections as compared with landmark-guided injections technique, (twelve randomized controlled trials were included in the meta-analysis). They found that intraarticular and periarticular injections using ultrasound guidance significantly improves the accuracy of joint injections.

In our study we found that PRP injection was more effective in mild to moderate OA than severe OA. This is in agreement with Patel $\boldsymbol{e t}$ al. ${ }^{\left({ }^{(32)}\right.}$ who concluded that a single dose of PRP is as effective as 2 injections to alleviate symptoms in early knee OA.

\section{CONCLUSION}

The application of ultrasound guided single dose PRP is a safe and effective method for treating all grades of knee OA mainly mild to moderate affection and periarticular pain generator structures aiming to reduce pain and improve knee function.
However, this study has some limitations. We did not include a control group receiving another form of injection (corticosteroids or hyaluronic acid). Also, a longer period of follow up is needed to confirm our results.

\section{Conflict of interest: none.}

Funding: This research did not receive any specific grant from funding agencies in the public, commercial, or not-for-profit sectors.

\section{REFERENCES}

1. Glyn-Jones S, Palmer A, Agricola $\mathrm{R}$ et al. (2015): Osteoarthritis. The Lancet, 2015: 376-387.

2. Lee C, Huang M, Chai C et al. (2008): The validity of in vivo ultrasonographic grading of osteoarthritic femoral condylar cartilage: a comparison with in vitro ultrasonographic and histologic grading. Osteoarthritis Cartilage, 16:352-358.

3. Friedman L, Finlay K, Jurriaans E (2001): Ultrasound of the knee. Skeletal Radiol., $30: 361-377$.

4. Klauser A, Tagliafico A, Allen G (2012): Clinical indications for musculoskeletal ultrasound: a delphi-based consensus paper of the European society of musculoskeletal radiology. Euro Radiol., 22: 1140-1148.

5. Jackson D, Evans N, Thomas B (2002): Accuracy of needle placement into the intra-articular space of the knee. J Bone Joint Surg Am., 84: 1522-1527.

6. Sibbitt W, Peisajovich A, Michael A et al. (2009): Does sonographic needle guidance affect the clinical outcome of intraarticular injections?. The Journal of Rheumatology, 36: 1892-1902.

7. Ibrahim D, El-Gazzar N, El-Saadany H et al. (2019): Ultrasound-guided injection of platelet rich plasma versus corticosteroid for treatment of rotator cuff tendinopathy: Effect on shoulder pain, disability, range of motion and ultrasonographic findings. The Egyptian Rheumatologist, 41: 157-161.

8. Smink A, Van Den Ende C, Vliet T et al. (2011): Beating osteoarthritis: Development of a stepped care strategy to optimize utilization and timing of non-surgical treatment modalities for patients with hip or knee osteoarthritis. Clin Rheumatol., 30:1623-1629.

9- Gasparyan YA, Ayvazyan L, Pretorius E et al. (2014): Platelets in rheumatic diseases: friend or foe?. Current Pharmaceutical Design, 20: 552-566.

10. Razaq S, Ejaz A, Rao $S$ (2015): The role of intraarticular platelet rich plasma (PRP) injection in patients with internal knee derangements. J Coll Physicians Surg Pak., 25: 699-701.

11. Alsousou J, Ali A, Willett K (2013): The role of plateletrich plasma in tissue regeneration. Platelets, 24: 173-182.

12. Altman R, Asch E, Bloch D (1986): The American college of rheumatology criteria for the classification and reporting of osteoarthritis of the knee. Arthritis Rheum., 29: 1039-1049.

13. McCormack H, Horne D, Sheather S (1998): Clinical applications of visual analogue scales: a critical review. Psychol Med., 18: 1007-1019. 
14. Boone DC, Azen SP (1979): Normal range of motion of joints in male subjects. Journal Bone Joint Surg AM., 61:75675-9.

15. Guermazi M, Poiraudeau S (2004): Translation, adaptation and validation of the Western Ontario and McMaster Universities osteoarthritis index (WOMAC) for an Arab population: the Sfax modified WOMAC. Osteoarthritis Cartilage, 12: 459-468.

16. Collins NJ, Misra D, Felson DT (2011): Measures of knee function: International Knee Documentation Committee (IKDC) Subjective Knee Evaluation Form.Arthritis Care Res., 63: 208-228.

17. Catay E, S. Ruta S, Rosa J et al. (2012): Ultrasound (US) findings in patients with knee pain: sensitivity and specificity for the diagnosis of knee osteoarthritis and development of an us prediction score. Arthritis \& Rheumatism, 64: 9-14.

18. Kellgren JH and Lawrence JS (1957): Radiological assessment of osteo-arthrosis. Ann Rheum Dis., 16: 494502.

19. Peck E, Mautner K (2014): Rehabilitation after plateletrich plasma injections for tendinopathy. In: Platelet-Rich Plasma. Springer.

20. Filardo G, Buda R, Timoncini A (2011): Platelet-rich plasma intra-articular knee injections for the treatment of degenerative cartilage lesions and osteoarthritis. Knee Surg Sports Traumatol Arthrosc., 19: 528-535.

21. Filardo G, Kon E, Vaccaro F (2012): Platelet-rich plasma intra-articular injections for cartilage degeneration and osteoarthritis: Single versus double-spinning approach. Knee Surg Sports Traumatol Arthrosc., 20: 2082-2091.

22. O'Connell B, Wragg NM, Wilson SL (2019): The use of PRP injections in the management of knee osteoarthritis. Cell Tissue Research, 19:1-10.

23. Lawrence RC, Felson DT, Helmick CG (2008): Estimates of the prevalence of arthritis and other rheumatic conditions in the United States. Part II. Arthritis Rheum., 58: 26-35.

24. Felson DT, McAlindon TE, Anderson JJ et al. (1997): Defining radiographic osteoarthritis for the whole knee. Osteoarthritis Cartilage., 5: 241-250.

25. Arden N, Nevitt MC (2006): Osteoarthritis: epidemiology. Best Pract Res Clin Rheumatol., 20: 3-25.
26. Vasilevska V, Szeimies U, Sta“bler A (2009): Magnetic resonance imaging signs of iliotibial band friction in patients with isolated medial compartment osteoarthritis of the knee. Skeletal Radiol., 38: 871-875.

27. Brage ME, Draganich LF, Pottenger LS (1994): Knee laxity in symptomatic osteoarthritis. Clin Orthop Relat Res., 304: 184-189.

28. Filardo G, Buda R, Timoncini A et al. (2011): Plateletrich plasma intra-articular knee injections for the treatment of degenerative cartilage lesions and osteoarthritis. Knee Surg Sports Traumatol Arthrosc., 19:528-35.

29. Iaccarino M, Borg-Stein J (2016): Platelet-Rich Plasma in Knee Osteoarthritis in the Athlete. In Platelet Rich Plasma in Musculoskeletal Practice. Springer, London.

30. Kon E, Mandelbaum B, Buda R (2011): Platelet-rich plasma intra-articular injection versus hyaluronic acid visco- supplementation as treatments for cartilage pathology: from early degeneration to osteoarthritis. Arthroscopy, 27: 1490-1501.

31. Gosens T, Den Oudsten B, Fievez E et al. (2012): Pain and activity levels before and after platelet-rich plasma injection treatment of patellar tendinopathy: a prospective cohort study and the influence of previous treatments Int Orthop., 36: 1941-1946.

32. Patel S, Dhillon MS, Aggarwal S et al. (2013): Treatment with platelet-rich plasma is more effective than placebo for knee osteoarthritis: a prospective, double-blind, randomized trial. Am J Sports Med., 41: 356-364.

33. Gobbi A, Karnatzikos G, Mahajan V et al. (2012): Platelet-rich plasma treatment in symptomatic patients with knee osteoarthritis: preliminary results in a group of active patients. Sports Health, 4: 162-172.

34. Paterson KL, Nicholls M, Bennell K et al. (2016): Intraarticular injection of photo-activated platelet-rich plasma in patients with knee osteoarthritis: a double-blind, randomized controlled pilot study. BMC Musculoskelet Disord., 17:67-69.

35. Huang Z, Du S, Chen G et al. (2015): Effectiveness of ultrasound guidance on intraarticular and periarticular joint injections: systematic review and meta-analysis of randomized trials. Am J Phys Med Rehabil., 94: 775-783. 